\title{
Estrategias de intervención en organizaciones civiles. Las organizaciones de mujeres
}

\author{
Intervention strategies in civil society organizations: \\ women's organizations \\ Gloria Jovita Guadarrama-Sánchez / gguadarr@cmq.edu.mx \\ orcid.org/0000-0003-3726-5646 \\ El Colegio Mexiquense A.C., México \\ Esmeralda Pliego-Alvarado/ epliegoa@gmail.com \\ orcid.org/0000-0001-6178-4222 \\ Universidad Autónoma del Estado de México, México
}

\begin{abstract}
This article aims to explore the impact of the intervention strategies of civil organizations for the attention of women in Mexico in producing a particular vision on gender and the assessment of their performance. The analysis is supported on an intervention typology, an assessment of performance and on the information from three study cases. Some findings show that the intervention strategies condition some of the assessed elements and impact on the differentiation of the women's involvement from gender perspective.
\end{abstract}

Key words: intervention strategies, women's civil organizations, gender perspective, organization's performance.

Resumen: El artículo está dirigido a explorar cómo inciden las estrategias de intervención de organizaciones civiles para la atención de las mujeres en México, en la conformación de una visión particular sobre el género y en la valoración de su desempeño. El análisis se sustenta en la elaboración de una tipología de intervención, una evaluación de desempeño y en la información aportada por tres estudios de caso. Algunos hallazgos muestran que las estrategias de intervención condicionan algunos de los elementos evaluados e inciden en la diferenciación del involucramiento de las mujeres desde la perspectiva de género.

Palabras clave: estrategias de intervención, organizaciones civiles de mujeres, perspectiva de género, desempeño de las organizaciones. 


\section{Introducción}

A partir del reconocimiento de que las organizaciones de la sociedad civil que trabajan a favor de las causas de las mujeres tienen capacidades para modificar las condiciones que obstaculizan su desarrollo, se propone destacar su importancia en la integración de una visión sobre el género, el tipo de estrategias empleadas como prototipos operacionales. Se parte del supuesto de que los cursos de acción elegidos al tiempo que inciden en el logro de los objetivos y en el desempeño organizacional, modelan la identidad y características de las organizaciones y construyen una representación sobre las formas de atender los problemas que les afectan.

Se advierte que en los últimos años se ha dado un vuelco en los enfoques sobre el género, al mostrar el incremento de los actores femeninos no sólo como víctimas o sujetos de atención, sino como agentes que intervienen y diseñan las estrategias para atender a otras mujeres. En este sentido, es necesario conocer: ¿quiénes son los actores que diseñan, planean y aplican las acciones?, ¿cómo surgen las organizaciones?, ¿cómo inciden en la solución de los problemas?, ¿cómo participan las mujeres en los propósitos de la organización?

Para responder esos cuestionamientos, se presentan tres estudios de caso de organizaciones civiles que atienden mujeres en el estado de Chiapas: Fundación Cántaro Azul A.C., Marie Stopes A.C. y Hogar Comunitario Yach'il Antzetic A.C.. El análisis se estructura en campos que comprenden los aspectos funcionales, organizacionales y la relación de la organización con su entorno. La configuración de prototipos operativos como estrategias se realizó con el apoyo de entrevistas a profundidad llevadas a cabo con los principales agentes de las organizaciones, además de la consulta documental y de recursos electrónicos de cada organización.

Con esos elementos se exploran aspectos como la percepción de los integrantes de la organización sobre su papel en mejorar las condiciones de la mujer y los cambios en la participación de las mujeres involucradas con los programas. Para vincular las estrategias con el desempeño, se aplicó el instrumento Protocolo para la Evaluación de Asociaciones ${ }^{1}$ (Puga y Luna, 2012) a los miembros de las organizaciones. ${ }^{2}$

1 El instrumento de evaluación aplicado a integrantes de las organizaciones de mujeres es un cuestionario que realizó la Red de Estudios para el Desarrollo Asociativo (REDA), publicado por el Instituto de Investigaciones Sociales de la UNAM en 2012; es un documento público de acceso abierto, a través de la versión electrónica del libro (Puga y Luna, 2012).

2 En una primera etapa se realizó trabajo de campo para conocer e identificar a las 
El texto se divide en tres apartados: el primero resume los cambios que han registrado históricamente las estrategias de las organizaciones de mujeres en nuestro país y su relación con la visión de género; el segundo describe los casos de estudio, su contexto y los resultados de su desempeño; el tercero vincula las estrategias de intervención con el desempeño de las organizaciones en las esferas que contempla el instrumento: funcional, organizacional y en su relación con el entorno.

Se argumenta que la estrategia define y diferencia a las organizaciones, modelando una visión particular de la perspectiva de género y que tales prácticas tienen una influencia recursiva en la estructura organizacional. Asimismo, se considera que las estrategias se definen por su origen, por los actores que las diseñan e instrumentan, y por el nivel de la intervención.

\section{De organizaciones para mujeres a organizaciones de mujeres}

Es reconocido que las personas participan en las asociaciones civiles para lograr, juntos, objetivos que de otro modo serían difíciles de conseguir y que en esos empeños, dependiendo de sus fines, es posible generar bienes sociales. Así, se observa a las organizaciones de mujeres como agrupaciones con fines, funciones, estructuras y cursos de intervención específicos que las diferencian en el mundo social. La mirada lleva implícito que, de modo general, sus intenciones estarán dirigidas a lograr avances en las condiciones de equidad, igualdad de oportunidades y vigencia de derechos para las mujeres. En esa percepción un tanto idealizada del actuar de las organizaciones, no se deja de lado que las formas de participación y la visión que subyace en ellas se han modificado de acuerdo con las transformaciones de la vida económica, política y social.

Sabemos que en el transcurso del tiempo y de modo diverso el papel que juegan las mujeres en el establecimiento del orden social ha transitado de ser agentes pasivos, hacia ser agentes de su propio desarrollo. En ese proceso podemos ubicar la influencia de las corrientes de movimientos feministas y el papel que han jugado las mujeres dentro de estos movimientos.

Como lo señala Espinosa (2009), en México, la forma que adoptaron fue en principio realizar reuniones de mujeres que revisaban sus situaciones

organizaciones y seleccionar las participantes en el estudio, con base en que contaran con una forma distintiva o modelo de intervención; en una segunda etapa se realizaron entrevistas individuales a las(os) integrantes de las organizaciones para identificar el tipo de intervención; en la trecera etapa se aplicó el instrumento de evaluación. La información recabada fue codificada y calificada por integrante y por organización, de acuerdo al formato validado y registrado en el protocolo del instrumento evaluativo. 
de opresión individual; con el paso del tiempo, y al identificar coincidencias, se reconoció que se trataba no sólo de problemas personales, sino de problemas sociales; así, se comenzaron a integrar círculos de estudio e investigación, células de partidos políticos, grupos editoriales y núcleos de profesionales para proporcionar servicios específicos.

Se intentaba articular una agenda que incluyera una política feminista, en donde la igualdad política y legal para las mujeres, el derecho al trabajo y a la independencia económica, el derecho a decidir sobre su cuerpo y el pleno ejercicio de la sexualidad fueran un primer acercamiento a la igualdad de género (Espinosa, 2009). Más tarde, surgieron nuevas formas de intervenir, no sólo para satisfacer necesidades básicas, sino buscando participación política real, incursión en actividades antes destinadas a los varones, rescatando y repensando el papel de las mujeres en la sociedad.

Es así como en los años ochenta, las formas organizativas de las mujeres constituirán dos vertientes del movimiento feminista; por un lado, el feminismo popular, integrado por organizaciones de mujeres de barrios pobres de las urbes, por grupos de obreras y empleadas y por campesinas que entrelazaron sus luchas gremiales, sociales y políticas; y por otro, el feminismo civil, integrado por organizaciones civiles, también llamadas no gubernamentales (ONG) (Espinosa, 2009).

Con el feminismo popular, se buscaba la movilización de las mujeres en organizaciones mixtas, la casa y la familia, el centro de trabajo, la comunidad rural o urbana y las organizaciones sindicales, campesinas y vecinales. Con el feminismo civil, la participación de la mujer tuvo otras connotaciones, las organizaciones buscaron incidir en los movimientos sociales, pero también ser una fuerza con personalidad y objetivos propios, con identidad y proyectos para las necesidades específicas de las mujeres, siempre considerando la igualdad de género (Espinosa, 2009).

En ese marco se visibilizan tendencias en cuanto a la adscripción de las mujeres en los movimientos, organizaciones y formas de intervención social: aquellas amplias y menos estructuradas, cuya expresión se da por medios de movimientos sociales; y otras que dan un paso hacia la institucionalización de las prácticas, como las organizaciones civiles (OSC). En adelante solamente se hablará de las mujeres organizadas en asociaciones civiles, su impacto en problemas sociales concretos referidos a sus condiciones de bienestar y a la posición que ocupan las mujeres en la acción de las organizaciones. 


\section{Las organizaciones de mujeres}

Las organizaciones de mujeres que se constituyeron como asociaciones civiles fuertes fueron pocas. De manera general se pueden encontrar tres vertientes de su participación: 1) aquellas conformadas por mujeres que buscaron una reivindicación de género y que surgieron desde las filas feministas; 2) las asociaciones surgidas desde la beneficencia social, la filantropía y el voluntariado ligado a movimientos religiosos; y 3) las que surgieron de la vertiente popular, donde las mujeres actúan organizadas para resolver problemas colectivos (Eurosur, 2015).

En 1975, se confeccionó un primer registro de organizaciones de mujeres, principalmente asistenciales, seguidas por otras con objetivos gremiales, de apoyo a la cultura y otras de carácter político. En ese mismo año, se identificaron otros tipos de organizaciones: el $11 \%$ eran de género, el $11.4 \%$ de voluntariado, el 5.2\% de esposas, el $2.9 \%$ por residencia, el $2.4 \%$ de inmigrados, el 6.7\% culturales y el 38.6\% de beneficencia (Valdés, 1995).

En ese mapa es difícil identificar líneas de acción feministas, ya que, muchas veces, las participantes no se reconocían como tales, compartían valores asociados con los procesos reivindicativos de género, aunque sus motivaciones expresas tendieran hacia lo religioso o a lo profesional, como se considera que sucedía.

Otros rasgos eran que estaban constituidas por pequeños grupos, la mayoría no rebasaban diez integrantes; su intervención se centraba en la formación de líderes, capacitación y asesoría legal (26\%); en acciones ligadas a la sobrevivencia (22\%), en menor medida a las actividades académicas (18.6\%), muy pocas atendían la violencia hacia las mujeres (16\%), y la defensa de los derechos humanos y sindicales (14\%) (Tuñon, 1997: 58). Actualmente, con factores innovadores como las tecnologías de la información, la facilidad de movilización y nuevas estrategias de gestión de recursos, las organizaciones han ampliado sus intencionalidades.

En esos datos es posible notar que han sido procesos perfilados históricamente los que han permitido que las formas de participación y las estrategias de intervención se diversificaran, encontrando nuevos nichos de atención a las mujeres, desde las básicas como dotación de alimentos, hasta los prototipos contemporáneos que buscan el desarrollo de capacidades y el empoderamiento. 


\section{Las estrategias de intervención}

La estrategia de intervención es una vía organizativa y operativa que combina diferentes elementos, a través de los cuales las acciones relativamente institucionalizadas de las organizaciones marcan perfiles de actuación que imprimen una dirección a sus acciones; en este sentido, la estrategia de intervención busca relacionar a la población objetivo de un proyecto con los objetivos y fines de la organización (Rofman y Villar, 2006).

Es importante advertir las distintas formas en las que las estrategias de intervención son determinantes de los resultados obtenidos por las organizaciones. La exploración que se presenta se efectúa considerando la posición que ocupan las mujeres en la estrategia y la visión de género desde la cual se construye el curso de la acción.

En ese contexto se pueden reconocer distintos esquemas de relación entre los actores de la organización: a) unidireccional, cuando existe un proveedor y un receptor del apoyo; b) recíproco, cuando se realiza en términos que imponen obligaciones de cooperación, intercambio y reciprocidad, que llevan implícito el hecho de compartir entre los miembros de la organización y sus beneficiarios los recursos que resultan de su cooperación. Se aporta y se recibe (aunque probablemente no en la misma medida en que se contribuye); c) grupal, cuando se dirige hacia grupos específicos, lo cual implica criterios de focalización (niños, ancianos, madres solteras, campesinos, etc.); d) comunitario, cuando las intervenciones afectan los roles y generan cambios estructurales en las comunidades; por ejemplo, la creación de una asamblea comunitaria con inclusión de las mujeres. ${ }^{3}$

\section{Organizaciones y tipos de intervención}

Aunque cada organización genera en el transcurso del tiempo sus propios mecanismos de intervención, se distinguen en los esquemas relacionales que les dan consistencia tendencias que pueden construir tipologías de intervención. Para este caso se han identificado tipos ideales, que si bien son útiles para los propósitos del análisis, en realidad son rasgos obtenidos del entorno donde se mueven las organizaciones y se encuentran frecuentemente combinados; por ello se parte de reconocer que no se hallan de manera pura.

3 La formulación de las líneas de relación y la configuración de los grupos a los cuales se dirige la acción de las organizaciones está basada parcialmente en las propuestas de Gottlieb (1988) y de Gracia (1997). 
La tipología se realizó luego de un trabajo empírico, se buscó que la población objetivo fueran las mujeres o que sus programas incidieran directamente en ese sector; y que fueran organizaciones estructuradas bajo la figura legal de asociación civil, lo cual podía asegurar que no tuviesen fines declarados de lucro; asimismo, la selección tomó en cuenta que su impacto social fuera reconocido por las destinatarias de sus acciones.

En este marco, encontramos tres tipos de interacción que estructuran la intervención que llamaremos: desde fuera, participante y desde dentro (véase Cuadro $\left.1^{4}\right)$. En el primer tipo, la intervención se configura desde una visión que considera la existencia de una población que necesita apoyos, y las mujeres, como sector vulnerable o víctimas individuales, son consideradas sujetos de atención. En este tipo de intervención, el involucramiento de la organización puede ser menor, y sus integrantes no son parte de la población objetivo.

En el segundo tipo, se percibe a la población objetivo (las mujeres) como agentes de su propia transformación y se desarrollan estrategias para, con sus aportaciones, generar ideas y solucionar sus problemas. El involucramiento de la organización se ancla en la identificación compartida de los problemas; sus estrategias se relacionan con la acción participativa o la intervenciónacción, como una variante de la investigación acción. ${ }^{5}$

En el tercer tipo, la interacción refiere a organizaciones cuyos integrantes son o han sido parte de la población objetivo. Esto se puede observar cuando, por ejemplo, se atiende a mujeres, madres solteras, y ellas tuvieron esa condición. La intervención se ancla en las experiencias o vivencias compartidas de los problemas a resolver. Este tipo de intervención tiene que ver con el modelo de investigación participante natural.

\section{Componentes y perfiles de intervención}

Para la integración de la tipología, a partir de la percepción de los integrantes de las organizaciones sobre las relaciones se identificaron siete componentes: 1) pertenencia e identificación con la población objetivo;2) percepción sobre el rol de los participantes y la población objetivo; 3) planeación de la acción;

4 Los cuadros y las gráficas se encuentran en el Anexo, al final del presente artículo (Nota del editor).

5 Kurt Lewin menciona la investigación acción (Lacouture, 1996) como un ejercicio colectivo, autorreflexivo, generando dialéctica entre teoría y práctica. El concepto se ha nutrido y aplicado en distintos campos como en las organizaciones comunitarias y en la investigación social. 
4) mecanismos de control y sanción; 5) espacio de actuación; 6) temporalidad del impacto de la intervención; 7) nivel de intervención. La combinación de los componentes configura los perfiles específicos de intervención.

La percepción sobre la intervención es entendida como el conjunto de nociones identitarias que son reconocidas, aceptadas y asumidas en común por las personas integrantes de la organización y las relaciones que establecen con los integrantes de la comunidad a la cual dirigen sus esfuerzos; conlleva que los actores compartan los mismos códigos y lenguaje, mejorando los procesos de comunicación y generando empatía, lo cual facilita establecer lazos de confianza con la comunidad o población objetivo.

En ese sentido, si bien la delimitación del campo de actuación señala que la población objetivo son las mujeres, el segundo componente se dirige a distinguir el carácter de su participación como activa o pasiva; es decir, si generan acciones propias tendientes a cambiar sus condiciones, o si, en cambio, se perciben como receptoras o destinatarias de apoyos o prestaciones.

La planeación se relaciona con la forma en que se conciben los programas y proyectos que se aplican en las comunidades y grupos; implica diferenciar si la población objetivo se involucra desde el diseño y concepción de la estrategia, si se va integrando a lo largo de su instrumentación o solamente participa en la etapa de aplicación. El criterio implícito es que a mayor involucramiento de las personas beneficiarias, mayor será la aceptación de las acciones dentro de la comunidad.

Los mecanismos de control y sanción refieren a las vías establecidas para la salida, vigilancia y castigo en el caso de no cumplir con las reglas formales e informales de la organización. En el análisis sólo se considera su procedencia, buscando identificar si provienen o son establecidos por entidades externas, si se establecen mediante acuerdos internos o responden al consenso entre los actores intervinientes. Es decir, se considera, en el caso de violentar las reglas, a quién o quiénes corresponde decidir las sanciones; pues al parecer las personas tienen mejor disposición a afrontar las consecuencias cuando intervinieron en establecer las normas; a la vez, existe una menor aceptación en el caso de que sean reglas externas o percibidas como mecanismos impositivos.

El ámbito de actuación es clave, pues tiene que ver con la cohesión de los grupos, si la organización se configura como un ámbito estructurado institucionalmente con niveles jerárquicos y operativos que funcionan con base en reglas, como algunas organizaciones educativas; o si son semiestructurados, como las organizaciones de danzantes locales; o en caso de ser móviles y poco institucionalizados, como los jóvenes en y de la calle. Se asume que hay más posibilidades de intervenir en grupos formalizados, y existen mayores difi- 
cultades para acceder y participar con grupos abiertos, donde se depende de decisiones individuales para integrarse, recibir o intervenir activamente en los propósitos de la organización.

El sexto componente contempla la temporalidad del impacto de las intervenciones, si son duraderas como la alfabetización, si son resultados de efecto inmediato o a corto plazo y sin seguimiento como la aplicación de una vacuna; si se trata de cubrir una necesidad básica, o su incidencia es a mediano o largo plazo (salud, educación, desarrollo de habilidades). El séptimo componente captura la forma y nivel de la interacción entre la organización y su población objetivo, y si se circunscribe a individuos, grupos o comunidades.

\section{Estrategias de intervención y evaluación del desempeño de las organizaciones}

Las intervenciones cobran significado cuando se observan y valoran los resultados de su acción en la generación de bienes sociales, éstos pueden variar dependiendo de lo que se evalúe. Natal (2010) advierte que existen cuatro enfoques predominantes para evaluar el desempeño: 1) la organización cumple los objetivos para los que se creó; 2) cuenta con recursos financieros suficientes y es sustentable; 3) ha logrado un impacto en la población más allá de sus objetivos; 4) tiene la capacidad de adaptarse al entorno. De ello se deriva la presunción de que la forma como se evalúa es condicionante de los resultados y existen tantas variantes de desempeño como formas de evaluación del mismo.

Una de las maneras de aproximarse a la estimación del desempeño es utilizando algún instrumento que permita visibilizar y cualificar los componentes y resultados de la intervención como bienes sociales; este es el propósito de la herramienta desarrollada y validada por la Red de Estudios sobre Desempeño Asociativo (REDA), denominada Protocolo para la evaluación de asociaciones que considera la complejidad de las configuraciones asociativas como principio clasificatorio, y desarrolla la evaluación de su desempeño mediante tres ejes o líneas de valoración: desempeño práctico, desempeño organizativo y la relación de la organización con el entorno (Puga y Luna, 2012). ${ }^{6}$

6 Dada la heterogeneidad de las asociaciones civiles, es necesaria una clasificación inicial que permita estimar de manera distinta asociaciones que constitutivamente son diferentes. La complejidad como criterio de clasificación inicial es entendida como la combinación de cuatro propiedades: autonomía política, autonomía individual de sus miembros, interdependencia y dinamismo. La combinación de estas propiedades se mueve en una línea que va de las asociaciones de muy alta complejidad a las de muy baja complejidad. La mayor o menor complejidad se determina a partir de ciertas características de la asociación relativas a su tipo de membresía, formas de ingreso y permanencia, formas de organización interna, así como a las características de sus objetivos y recursos (Puga y Luna, 2012: 14). 
Se integra por dos cuestionarios y dos fichas: Cuestionario de Colocación (CC): permite situar a la organización de acuerdo con su nivel de complejidad; el Cuestionario de Evaluación del Desempeño (CD): evalúa los ejes antes mencionados; la Ficha Individual (FI): sirve como instrumento de información complementaria; la Ficha de la Asociación (FA): permite tener una visión más completa y global de la organización. Con los elementos que proporciona, en una vertiente, el esquema preliminar de la tipología de intervención, y en la otra, el instrumento de evaluación de desempeño, se examinan, en los apartados siguientes, las estrategias de intervención.

\section{Tres organizaciones de mujeres y para mujeres: sus estrategias de intervención}

Las organizaciones que integran este estudio son: Marie Stopes A.C. (MS), Fundación Cántaro Azul A.C. (FCA) y Hogar Comunitario Yach'il Antzetic A.C. (HCYA), las cuales fueron seleccionadas porque poseen rasgos que las circunscriben a un campo de actuación común y, simultáneamente, porque tienen características diferenciales de complejidad y actuación que posibilitan atribuirles perfiles propios para cualificar sus estrategias, pues se relacionan con la salud, ámbito donde el involucramiento de las mujeres y el rol que desempeñan en la organización son claves para el modo como asumen los fines de la salud reproductiva, sexual y general, utilizando diferentes formas de intervención para atender su población.

Asimismo, las organizaciones elegidas cuentan con características comunes: a) la integración femenina de sus miembros; b) la población objetivo focalizada en el sector mujeres; y c) comparten un espacio de actuación comunitaria, identificado como su inserción, pertenencia o involucramiento con comunidades de población indígena.

En cuanto a la composición de su población objetivo, las tres atienden a otras mujeres; en el caso de FCA aun cuando la mayoría de las acciones van encaminadas a las mujeres, los beneficios son compartidos con la comunidad. Finalmente, las tres organizaciones laboran en San Cristóbal de las Casas; en los casos de FCA y MS, la intervención se realiza en comunidades indígenas de manera directa (los miembros del equipo se trasladan a trabajar a las comunidades); HCYA, por su parte, recibe en sus instalaciones a mujeres de comunidades indígenas.

Las organizaciones presentan también diferencias y contraposiciones. En cuanto a sus fines y objetivos, si bien éstos se enmarcan en la procuración de la salud, mientras que en HCYA -con orígenes históricos religiosos- se 
busca que las mujeres tengan hogar para tener a sus hijos y cuidarlos, por su parte MS deja abierta la opción del aborto, si las condiciones y la voluntad de la mujer lo permiten. En tanto, en FCA la finalidad es el mejoramiento de la calidad del agua para consumo humano; pero para conseguirlo trabaja, especialmente, con el empoderamiento y cohesión social de los grupos de mujeres que forman parte de los programas. En torno a esos puntos se marcan en el análisis las diferencias entre las organizaciones.

Para cualificar las estrategias de intervención -que son el eje central del análisis-, se reconoce de inicio que tienen tendencias visibles hacia los prototipos descritos en el apartado anterior: HCYA posee inclinación al modelo Desde dentro, MS cuenta con elementos que se inclinan al modelo Desde fuera, y FCA posee características mixtas o del modelo Participante. A su vez, su desempeño es estimado mediante la evaluación que se analizará más adelante. Cabe destacar que se eligieron estas tres asociaciones por asumir una visión particular de género, por su búsqueda para mejorar la salud de las mujeres desde distintos enfoques, y tener estrategias de intervención diferenciadas, lo cual ayuda a explicar la propuesta tipológica.

\section{Marie Stopes, A.C.}

Marie Stopes México A.C. forma parte de un programa de Marie Stopes International, organización mundial no gubernamental líder en salud sexual y reproductiva, que opera bajo el modelo social business, ${ }^{7}$ con estrategias de intervención en las cuales mujeres de zonas alejadas pueden tener acceso a sus servicios; la organización interactúa con su población objetivo en procesos de educación y de salud sexual, previniendo embarazos no deseados e infecciones de transmisión sexual.

Su misión es clara: "Hijos por elección no por azar" y también su visión: "Ser la principal y mejor organización de planificación familiar en el mundo, haciendo una diferencia sostenible” (Marie Stopes, México, 2014). A lo largo de los años, la organización - pionera en el control de la natalidad- ha tenido presencia en zonas alejadas de recursos económicos escasos. En 1921, su primera clínica de planificación familiar fue fundada por Marie Stopes en Inglaterra, y en 1972, se creó Marie Stopes International, como organización con presencia en 41 países. En 1999, llegó a México con el establecimiento

7 El Instituto Tecnológico de Massachusetts lo define como "una actividad que usa los medios sociales, el software social y las redes sociales para permitir conexiones mutuas más eficientes y efectivas entre personas, información y recursos (Sloan Management Review, 2012, citado por Fuetterer, 2013). 
del primer Centro de Salud Sexual y Reproductiva, en San Cristóbal de las Casas, Chiapas; y en 2005, abrió otro centro en la misma entidad. Amplió su cobertura y creó nueva infraestructura en Bochil y Ocosingo, Chiapas, y en Juchitán, Oaxaca, cuando en el Distrito Federal se despenalizaba el aborto durante las primeras 12 semanas de gestación (2007). ${ }^{8}$

En ese mismo año se fundó el programa Parteras en Chiapas, en el cual se centra este análisis, con el equipo que realiza actividades desde San Cristóbal de las Casas, Chiapas. El programa Parteras de MS es una iniciativa que busca contribuir a la salud sexual y reproductiva de la población chiapaneca, y ha incidido principalmente en zonas indígenas de la región; consiste en dar un acompañamiento a las parteras locales, se les capacita y se llevan a cabo encuentros donde se comparten experiencias.

La evaluación de desempeño fue aplicada a los seis miembros del equipo del programa. Los resultados muestran que el índice de complejidad, en una escala de 0 a 10, es de 3.57, lo cual la ubica en baja complejidad. La calificación global de desempeño es de 6.75 , con un desempeño práctico o funcional de 6.8, desempeño organizativo de 6.5 y la relación con el entorno de 6.9; lo cual implica que su desempeño se encuentra dentro de los parámetros promedio previstos por la escala de evaluación (entre 3.0 y 7.0). De manera general, los aspectos evaluados presentan calificaciones aceptables en el marco esperado (véase Gráfico 1). ${ }^{9}$ Los datos ubican a la organización MS en un rango de complejidad baja y desempeño medio.

A su vez, las entrevistas amplias y semiestructuradas al personal de la asociación muestran que el perfil de intervención en el programa Parteras, tiene las siguientes características: a) buscan que las promotoras sean de las comunidades objetivo o con trayectoria de trabajo en campo, y por lo tanto, idealmente, tengan sensibilidad ante la problemática local; b) se capacita constantemente a las promotoras para poder intervenir en las comunidades así como a las parteras; $c$ ) se trata de que la ayuda sea lo más integral posible, dotando de asesoría jurídica y acercándose a las comunidades.

8 Resultado de dos reformas legislativas: 1) en 2002, se firma la iniciativa para legalizar el aborto en casos de malformación congénita o cuando está en riesgo la vida de la madre; 2) en 2003, la Asamblea Legislativa del Distrito Federal aprueba reformas al Código Penal y modifica la Ley de Salud para el Distrito Federal (Díaz-Olavarrieta et al., 2012).

9 De acuerdo con los parámetros generales del instrumento de evaluación las calificaciones menores a 3 o mayores a 7 deberían ser consideradas inusualmente bajas o excepcionalmente altas (Puga y Luna, 2012: 31) 
Gloria Jovita Guadarrama-Sánchez y Esmeralda Pliego-Alvarado. Estrategias de intervención en organizaciones civiles. Las organizaciones de mujeres

Fundación Cántaro Azul A.C.

Es una organización cuya misión declarada es mejorar la salud y la calidad de vida de las personas que viven en comunidades marginadas. Como visión general, mencionan que en cinco años generarán soluciones que beneficien de manera significativa y sostenible a más de un millón de personas en México y que contribuyan al enriquecimiento del sector del agua a nivel internacional. Trabajan con una metodología participativa con las comunidades, creando soluciones que consideran adecuadas al contexto donde laboran. Se encuentran vinculados con centros académicos, instituciones de gobierno, organismos internacionales, iniciativa privada y otras organizaciones de la sociedad civil con las que comparten objetivos o motivaciones (Fundación Cántaro Azul, 2015).

En el momento de la evaluación, el equipo estaba conformado por siete personas; aunque entre ellos se integran subgrupos que trabajan en distintos proyectos y colaboran de manera simultánea en todos. Se entrevistó a los siete integrantes. En el índice de complejidad se obtuvo 3.6 y una calificación global de desempeño de 7.4; el desempeño funcional fue de 8.3; el desempeño organizativo de 7.2; la relación con el entorno fue de 7.3 (véase Gráfico 2). La comparación de esos resultados con los parámetros del instrumento permite estimar a la organización con un nivel alto de desempeño general, en particular en lo correspondiente a su desempeño funcional.

En el perfil de la estrategia de intervención de Fundación Cántaro Azul, destacaron tres componentes: 1) los integrantes realizan estancias en comunidad donde con actividades y talleres logran conocer la problemática y ganarse la confianza de la población; puede llevar unas cuantas visitas o tardar meses en conseguir que las comunidades acepten la intervención; 2) el equipo ha buscado estar en constante capacitación, por lo tanto, sus integrantes han participado en cursos y recibido apoyos institucionales; 3) además de trabajar en comunidad, han logrado formar parte de redes académicas y de investigación, generando acciones de colaboración.

\section{Hogar Comunitario Yach'il Antzetic A.C.}

El Hogar Comunitario Yach 'il Antzetic A.C. se declara como un espacio donde se impulsan los derechos de las mujeres y niños sin discriminación (Hogar Comunitario, 2014). El obispo Samuel Ruiz y su hermana María de la Luz Ruiz García lo fundaron hace 19 años en la diócesis de San Cristóbal de las Casas. Yach 'il Antzetik significa "mujeres nuevas" en tzotzil; 
inicialmente recibía a mujeres de origen indígena, pero actualmente recibe a toda mujer que lo requiera.

En referencia al origen de la organización, se menciona que la hermana del padre Ruiz se dio cuenta del problema de violencia hacia las mujeres embarazadas y el consecuente abandono de recién nacidos, y consideró necesario atender esta situación que afectaba tanto a los niños como a las mujeres.

El obispo Samuel Ruiz ofreció ayuda a las mujeres abandonadas con embarazos no planeados y las ubicó en una casita al lado de la Catedral, donde las albergaba hasta que tenían el parto. Con el tiempo llegaron más mujeres y fue insuficiente el espacio; cuando el obispo pudo obtener recursos, al ver la actividad que continuaba su hermana, le donó la casa en 1997, para seguir ayudando a más mujeres, y ese mismo año se logró constituir como Asociación Civil.

Actualmente, se reciben de 50 a 100 mujeres al año -aun cuando el espacio con el que cuentan es para seis mujeres, las salas de parto también son utilizadas en caso de sobrecupo-, quienes proceden tanto de comunidades como de la ciudad, porque el problema de abandono y embarazo no planeado es generalizado; si bien sus acciones son impulsadas inicialmente por intenciones religiosas, no tienen preferencia por apoyar a mujeres católicas. En palabras de María:

En la asociación existe un reglamento donde se habla de respeto y de comprensión de la situación de las mujeres, la responsabilidad y el valor del trabajo. La mayoría están por amor al trabajo, si alguien requiere entrar, lo pueden hacer dependiendo del interés, se reciben voluntarias, y apoyo de una institución alemana con voluntariado. ${ }^{10}$

En el momento de la aplicación del cuestionario, la organización tenía cinco voluntarias y cuatro mujeres atendidas. Las colaboradoras se encontraban en rangos de edad de 20 a 40 años. Sus integrantes son en su totalidad mujeres, algunas con formación profesional como enfermería y psicología. La dirigencia es ejercida por una líder que actúa como responsable del equipo, pero cada persona tiene asignada una función, se elaboran informes y reflexiones sobre el trabajo en reuniones colectivas, conformadas por: dos enfermeras-parteras, una coordinadora de trama (trabajando con el arte manual), como tallerista está una psicóloga y alguien que se encarga de la producción de hongos setas.

La líder se elige en colectividad en una reunión y solo se cambia en caso de ser necesario. Las integrantes hablan tzotzil, tzeltal, tojolabal, español y proceden de comunidades distintas. No cuentan con un salario fijo, en ocasiones

10 Entrevista con María, encargada del Hogar en ese momento y tallerista (San Cristóbal de las Casas, Chiapas, 5 de diciembre de 2014). 
se labora sin recibir retribución. El Hogar Comunitario Yach 'il Antzetic recibe donativos particulares, tanto en especie (ropa, comida y otros) como en efectivo, y tienen vínculos con otras Asociaciones Civiles (Marie Stopes, Fundación León XIII, el dispensario médico indígena Esquipulas, el hospital regional), pero no del gobierno.

La forma de trabajo es por proyecto, y no se limita al parto y al cuidado previo, se imparten talleres productivos, de arte, empoderamiento de la mujer, autoestima, autocuidado, sobre cómo aprender a poner límites, seguridad personal y son abiertos al público. A las mujeres que se encuentran bajo su cuidado se les da apoyo psicológico y jurídico si así lo desean, incluso una vez terminada su estancia en la casa hogar.

En la evaluación del desempeño el grado de complejidad se fijó en 2.28, siendo el menor de los tres casos abordados, probablemente porque la totalidad de los recursos con los cuales cuentan son propios; existen objetivos claros y el voluntariado es constante, pues aun cuando no reciben pago por los servicios la presencia de voluntarias es relativamente permanente. La calificación global se ubicó en 6.8, clasificado en: desempeño funcional 6.9, desempeño organizativo 6.6 y relación con el entorno 7.0 (véase gráfico 3), este último dato se debe probablemente a la falta de vinculación con el gobierno.

En el perfil de la estrategia de intervención destacan tres componentes: 1) identificación y aceptación recíproca con la población destinataria de sus acciones; 2) retroalimentación en actividades; 3) vínculos con otras instituciones. Esto significa que si bien realizan campañas en redes sociales o físicas para darse a conocer, es la población objetivo quien se acerca a las instalaciones del hogar a pedir ayuda, sea por su ubicación territorial o porque se enteran de la asociación por otro vínculo.

Asimismo, una vez que se está dentro, se pueden llevar a cabo actividades que ayuden al Hogar Comunitario Yach 'il Antzetic a continuar con la labor; es decir, si se desea, las beneficiarias pueden permanecer más tiempo. Al realizar esas acciones, se aprovechan los vínculos con otras organizaciones civiles para apoyar en la recaudación, atender a las mujeres si se complica el parto, así como para brindar apoyo psicológico y jurídico.

\section{Análisis comparativo de los casos: desempeño asociativo y estrategia de intervención}

De acuerdo con la tipología de intervención y a las características encontradas en campo para cada una de las organizaciones, las estrategias se perfilan con las configuraciones siguientes: el Hogar Comunitario Yach 'il Antzetic A.C. 
se acerca al prototipo desde dentro, pues en su interacción con la comunidad es frecuente que las integrantes estén identificadas con los objetivos, por haber sido ayudadas por la misma organización. Las actividades son planeadas pensando en lo que ellas necesitaron en el momento en que estaban solas, embarazadas o con problemas de violencia doméstica. Aunque las decisiones están sujetas a la aceptación de todo el equipo, existe flexibilidad y, por lo tanto, el espacio de actuación es más o menos libre.

El perfil de intervención de Marie Stopes A.C., al ser una organización con bases internacionales y metodología de intervención clara y diseñada con modelos internacionales, se identifica con el modelo desde fuera, pues aunque buscan la generación de vínculos, las integrantes no forman parte de las comunidades donde intervienen, y el espacio de actuación es restringido; muchas de las acciones pueden ser a corto o mediano plazo.

Respecto a la Fundación Cántaro Azul A.C., se ubica en el prototipo participativo, debido a que los miembros del equipo que intervienen en las comunidades no son parte de éstas, pero trabajan en colaboración con ellas con metodologías participativas; incluso la vigilancia o acompañamiento que hace la organización es consensada comunitariamente; es decir, los parámetros de logro y las acciones son generadas cooperativamente. El equipo tiene elementos metodológicos, pero en la intervención surgen adecuaciones que ayudan a ajustarlos a las condiciones locales; se puede considerar que se manejan en un espacio semiestructurado, con acciones a mediano y largo plazo.

Las tres organizaciones son de baja complejidad, con objetivos, metas y funciones claras, la mayoría de los recursos son propios y los requisitos para el ingreso están predeterminados; las diferencias en la complejidad se concentran en la fluctuación y cantidad de voluntariado, la procedencia de los miembros y sus capacidades de adecuación a necesidades locales. En cuanto a su desempeño, las tres organizaciones obtuvieron calificaciones por encima del 6.5 (en una escala de 0 a 10); las diferencias se concentran en desempeño práctico funcional y en el desempeño asociativo (véase Cuadro 2).

La calificación global muestra que se encuentran en rangos con buen desempeño. El desempeño práctico o funcional se localizó entre 6.9 (MS) y 8.3 (FCA); esto es, el conjunto de las acciones que despliegan para cambiar el entorno donde operan es positivo, y los resultados que generan en términos de bienes sociales (servicios, símbolos, ideas, bienes y otros) son en general también positivos (Puga y Luna, 2012: 9).

En su desempeño organizativo, referido a la capacidad para coordinar las acciones de sus miembros, tomar decisiones, establecer acuerdos y procesar los conflictos internos, se obtuvieron resultados que van de 6.6 (MS) 
a 7.6 (HCYA). Este rubro está integrado por cinco criterios: reglas de decisión, procedimientos y mecanismos de decisión, integración, dirigencia y medios de observancia.

Los puntajes más altos se encontraron en el indicador de integración con la organización, con 9.5 (HCYA), 9.3 (FCA) y 7.8 (MS), lo cual se explica, en el caso del HCYA, por tener tendencias al modelo "desde dentro", donde los integrantes han generado un grado alto de identidad con la organización, condición que favorece asumir compromisos respecto a las metas logradas. De modo distinto, en el caso de MS, si bien se cuenta con el convencimiento acerca de los valores, metas y objetivos, el interés por participar se sustenta en la posibilidad de obtener un ingreso.

Los problemas detectados se centran en las reglas de decisión, ${ }^{11}$ con las calificaciones más bajas: 6.0 (HCYA), 6.1 (MS) y 7.0 (FCA), esto se explica en el hecho de que si bien existen reglas de decisión más o menos formalizadas, sobre todo en Yach 'il Antzetic, las reglas son construidas y reformuladas de acuerdo con la situación que se presenta, la cantidad de personas participantes y su disponibilidad para participar o no en actividades colectivas.

Finalmente, en la relación con el entorno, dimensión conformada por seis criterios: disponibilidad de capital, estrategias frente al desafío, interacción política, representación, relevancia social y legitimidad, las calificaciones más altas se obtuvieron en la disponibilidad de capital: HCYA con 7.7, FCA con 8.0 y MS con 75. Este rubro considera el capital social, económico y simbólico. Los datos más bajos se obtuvieron en relevancia social donde HCYA obtuvo 5.2, FCA, 4.8 y MS, 5.6.

Esto se justifica en la medida que las tres asociaciones cuentan con un capital estable, sobre todo respecto al capital social y económico, pues si bien no es suficiente en relación con la población potencial, sí logran encontrar fuentes estables de financiamiento. Dentro de las subcategorías que contempla la medición, el capital simbólico obtiene calificaciones menores, de 5.8 en HCYA, a 5.6 en las otras dos organizaciones, pues se requiere tiempo para lograr un capital simbólico y tiene que ver con el prestigio, conceptos que no fácilmente pueden reconocerse o describirse.

En cuanto a la visión de género, dados los objetivos y las acciones de cada organización se observa que: a) las tres organizaciones han sido conscientes de la desventaja en la cual se encuentran las mujeres respecto a los hombres en la comunidad, ya sea por maltrato físico, económico, sexual o por las restricciones

11 "Cuán apropiados y funcionales son tanto los métodos como los procedimientos de toma de decisiones y de formas de participación que tienen lugar dentro de la asociación" (Luna y Puga, 2012: 94). 
que enfrentan para la toma de decisiones sobre su propio cuerpo; b) existe el reconocimiento de que las mujeres pueden desarrollar capacidades novedosas o fortalecer las que ya se tienen; c) las organizaciones buscan la transformación de las condiciones actuales para mejorar la calidad de vida.

Sin embargo, al momento de emprender acciones, cada organización se distingue por una tendencia distinta en cuanto a la visión del papel de la mujer como agente de su desarrollo, y en este sentido se puede reconocer que: a) Marie Stopes A.C., como organización internacional, posee experiencia de intervención en comunidad, con tendencia hacia una visión de género que percibe a las mujeres como personas que necesitan ser ayudadas y cuyo papel es pasivo; b) Fundación Cántaro Azul A.C. posee características de intervención con un enfoque de género en el cual la mujer es parte activa de su propio desarrollo y, por lo tanto, es participante en cada etapa de los proyectos generados por la organización; c) Hogar Comunitario Yach 'il Antzetic A.C. si bien ubica inicialmente a las mujeres como personas que requieren ayuda, busca después que ellas sean parte de la organización y generen estrategias para hacerse de los recursos para mejorar su condición; respecto a su involucramiento, se inclinan hacia una estrategia combinada: pasiva inicialmente y activa en el momento de integrarse a la organización y atender a otras mujeres.

Se advierte que la evaluación del desempeño en las organizaciones de mujeres asume un carácter distinto al de otras organizaciones civiles, porque no solamente está ligada al logro de los objetivos de la organización, sino tiene repercusiones en la forma que asume la visión de género, así como en el tipo y el nivel de intervención; aspectos que son fundamentales por su incidencia diferente en la población objetivo. Cabe decir que resulta difícil hablar de buen desempeño, cuando la visión de género no considera a las mujeres como participantes activas en el desarrollo de sus capacidades, libertades y derechos.

Una advertencia sobre esta interpretación refiere a los cambios frecuentes que presentan las organizaciones tanto en sus formas constitutivas, como en las acciones que realizan. Aquí solamente se da cuenta de las características de las asociaciones en el momento específico en el cual se llevó a cabo el análisis, y actualmente, se han detectado cambios significativos en la estructura de las organizaciones examinadas, devenidos, por ejemplo, de la participación en proyectos más grandes que han diversificado sus acciones. No obstante esas limitaciones, la evidencia sobre la operación de las organizaciones de mujeres justifica la necesidad de abrir nuevos espacios en la evaluación de su desempeño; se requiere generar herramientas integrales de evaluación que tomen en cuenta las configuraciones de participación de la población objetivo. 
En apoyo de esa afirmación resulta útil observar la forma en la que a partir de estrategias distintas las organizaciones logran alcanzar sus metas; lo cual no es tarea fácil, pues se encuentra supeditada a condicionantes culturales arraigados en usos y costumbres y en las tradiciones comunitarias, presentes no sólo en comunidades indígenas, sino en las ciudades y localidades con desigualdades históricas. Asimismo, el ejercicio de evaluación ayuda a entender las oportunidades de actuación de las organizaciones que reflejan cambios en las formas en que la ciudadanía enfrenta los problemas, sus contradicciones y conflictos.

\section{Conclusiones}

El análisis de las organizaciones de mujeres vertido en estas páginas conduce a considerar que las organizaciones se constituyen como espacios de libertad e identidad, para compartir y actuar de manera colectiva sobre problemas individuales y que, simultáneamente, esas acciones tienen impacto como bienes sociales para el bienestar de grupos y comunidades.

Así, las organizaciones que tienen como población objetivo a otras mujeres construyen esquemas diferenciales de intervención, que inciden en su estructura interna, a partir del rol que asumen las mujeres como participantes en los fines de la organización. La premisa de que los cursos de acción elegidos modelan la identidad y características de las organizaciones adquiere sentido cuando se observa cómo, en las organizaciones donde las mujeres tienen un papel activo, su experiencia personal modela la forma y el curso de los emprendimientos. La organización no es entonces proveedora ajena, sino se vive como propia y la acción voluntaria se acentúa.

La recursividad, derivada de las estrategias con las que cada organización decide su intervención se concreta tanto en los procesos de transformación individual, como en la concepción del género, la orientación y la estructura de las organizaciones que adoptan las participantes, y en la conformación de niveles en el espacio social donde inciden las organizaciones. No se deja de lado el hecho de que pueden funcionar también como ámbitos reproductores del orden social que condiciona relaciones de subordinación y exclusión para las mujeres. No obstante, como se ha mostrado en el análisis, se visibilizaron tendencias afirmativas hacia desarrollar una visión de género que contempla el papel activo de las mujeres y el fortalecimiento de capacidades y poderes.

Es claro que la tipología preliminar propuesta tiene un correlato con el enfoque de género y que en ese carácter puede ser útil para explorar el papel de las mujeres en la estructura y funcionamiento de las organizaciones; pero 
también muestra las dificultades para la aplicación de cualquier tipología, ya que los roles de las mujeres se combinan y cambian en las diferentes etapas de aplicación de las estrategias. A pesar de esos obstáculos, la tipología posibilitó ubicar que en la intervención desde dentro el rol de las mujeres es más activo y se corresponde mejor con la visión moderna del género, que considera a la mujer agente de su propio desarrollo y la dota de capacidades de autogestión; pero se observa que los cambios en las comunidades fueron más fuertes en el caso de la intervención participante, pues busca no sólo la agencia femenina, sino la integración comunitaria. Por su parte, la intervención desde fuera también tiene influencia en la comunidad, porque es sistemática, estructurada, y genera mejoras considerables en la salud femenina, aunque menor en los procesos que generan la transformación de sus condiciones de dependencia o vulnerabilidad.

La relación entre la complejidad de las formas organizativas, el tipo de estrategia de intervención y el desempeño es menos evidente; de manera general, se puede estimar que el tipo de intervención participante genera mejor desempeño, y el tipo de intervención desde fuera es el de menor desempeño. En todos los casos, el ejercicio de evaluación ayuda a entender las oportunidades y los obstáculos que facilitan o constriñen el actuar de las organizaciones.

Vale la pena destacar, a manera de conclusión, que con estrategias distintas, las organizaciones de mujeres logran en mayor o menor medida impactos valiosos como bienes sociales, y la relevancia de fortalecer su participación impone ampliar el estudio de los perfiles de intervención y de evaluación.

\section{Referencias}

Díaz-Olavarrieta C. et al. (2012), "El Programa de Interrupción Legal del Embarazo en la Ciudad de México: experiencias del personal de salud", en Revista Panamericana de Salud Publica, núm. 32, vol. 6, EUA: Organización Panamericana de la Salud.

Espinosa, Gisela (2009), Cuatro vertientes del feminismo en México, México: Universidad Autónoma Metropolitana.

Eurosur (2015). Disponible en: http://www.eurosur.org/FLACSO/mujeres/mexico/orga1.htm [15 de diciembre de 2015].

Fuetterer, Stephan (2013), Social Business. Cómo la tecnología social y las personas contribuyen a la rentabilidad de las empresas. Disponible en: http://dksignmt.com/download/ Descargas/El\%20Libro\%20Del\%20Social\%20Business [15 de diciembre de 2015].

Fundación Cántaro Azul A. C. (2015). Disponible en: http://www.cantaroazul.org/ [15 de noviembre de 2014]. 
Gottlieb, B. H. (1988), "Support interventions: A tipology and agenda for research", en Duck, S.W. [ed.], Handbook of personal relationships, EUA: Wiley and Sons.

Gracia, E. (1997), El apoyo social en la intervención comunitaria, España: Paidós Ibérica.

Hogar comunitario Yach'il Antzetic A. C. (2014). Disponible en: http://yachilantzetic. org/es/mision [15 de noviembre de 2014].

Lacouture, Gerardo (1996), "El legado de Kurt Lewin”, en Revista Latinoamericana de Psicología, vol. 28, núm. 1, Colombia: Fundación Universitaria Konrad Lorenz.

Marie Stopes, México A.C. (2014). Disponible en: http://www.mariestopes.org.mx/ responsabilidad-social.html [17 de noviembre de 2014].

Natal, Alejandro (2010), "Modelo para el análisis y evaluación del desempeño asociativo", Ponencia en IX Congreso Internacional de Investigación sobre Tercer Sector, Estambul. Disponible en: https://c.ymcdn.com/sites/www.istr.org/resource/ resmgr/working papers_istanbul/natal_wp10.pdf [23 de mayo de 2015].

Puga, Cristina y Luna, Matilde [coords.] (2012), Protocolo para la evaluación de asociaciones, México: Universidad Nacional Autónoma de México, Instituto de Investigaciones Sociales, El Colegio Mexiquense. Disponible en: http://ru.iss.sociales.unam.mx/spui/ handle/IIS/4421 [19 de diciembre de 2016].

Rofman, Adriana y Villar, Alejandro [comps.] (2006), Desarrollo local. Una revisión critica del debate, Argentina: Espacio Editorial.

Tuñon, Esperanza (1997), Mujeres en escena: de la tramoya al protagonismo (1982-1994), México: Miguel Ángel Porrúa, UNAM-PUEG, Ecosur.

Valdés, Teresa (1995), Mujeres Latinoamericanas en cifras, México: Flacso.

\section{Entrevista}

María, encargada del HCYC, San Cristóbal de las Casas, Chiapas, 5 de diciembre de 2014. 


\section{Anexo}

\section{Cuadro 1}

Tipo de estrategia de intervención y componentes

\begin{tabular}{|c|c|c|c|}
\hline $\begin{array}{l}\text { Estrategia / } \\
\text { Componente }\end{array}$ & Desde fuera & Participante & Desde dentro \\
\hline $\begin{array}{l}\text { Pertenencia e } \\
\text { identificación }\end{array}$ & $\begin{array}{l}\text { No se es parte de la } \\
\text { población objetivo }\end{array}$ & $\begin{array}{l}\text { No se es parte pero } \\
\text { se reconoce a la } \\
\text { población objetivo }\end{array}$ & $\begin{array}{l}\text { Se es o se ha } \\
\text { sido parte de } \\
\text { la población } \\
\text { objetivo }\end{array}$ \\
\hline $\begin{array}{l}\text { La población } \\
\text { objetivo }\end{array}$ & $\begin{array}{l}\text { La población } \\
\text { objetivo se percibe } \\
\text { como un ente } \\
\text { pasivo }\end{array}$ & $\begin{array}{l}\text { Se ve a la población } \\
\text { objetivo como seres } \\
\text { activos y se colabora } \\
\text { con ellos }\end{array}$ & $\begin{array}{l}\text { La población } \\
\text { objetivo es } \\
\text { percibida como } \\
\text { agente de cambio }\end{array}$ \\
\hline $\begin{array}{l}\text { Planeación de la } \\
\text { acción }\end{array}$ & $\begin{array}{l}\text { Se planean desde } \\
\text { fuera y se aplican }\end{array}$ & $\begin{array}{l}\text { Se planean } \\
\text { colectivamente con la } \\
\text { población objetivo }\end{array}$ & $\begin{array}{l}\text { Se planean } \\
\text { desde dentro y se } \\
\text { aplican }\end{array}$ \\
\hline $\begin{array}{l}\text { Mecanismos de } \\
\text { control y sanción }\end{array}$ & Vigilancia externa & Vigilancia negociada & $\begin{array}{l}\text { Vigilancia } \\
\text { interna }\end{array}$ \\
\hline $\begin{array}{l}\text { Ámbito de } \\
\text { intervención }\end{array}$ & Estructurado & Semiestructurado & Libre \\
\hline Temporalidad & $\begin{array}{l}\text { Efectos de corto } \\
\text { plazo }\end{array}$ & $\begin{array}{l}\text { Efectos en el mediano } \\
\text { plazo o mixtos }\end{array}$ & $\begin{array}{l}\text { Efectos en el } \\
\text { largo plazo }\end{array}$ \\
\hline Nivel de & Individual & Grupal & Individual \\
\hline intervención & Grupal & Comunitario & Recíproco \\
\hline
\end{tabular}

Fuente: Elaboración propia con base en información de organizaciones seleccionadas. 
Gloria Jovita Guadarrama-Sánchez y Esmeralda Pliego-Alvarado. Estrategias de intervención en organizaciones civiles. Las organizaciones de mujeres

\section{Cuadro 2}

\section{Desempeño Asociativo en HCYA, FCA y MS}

\begin{tabular}{llll}
\hline \multirow{2}{*}{\multicolumn{1}{c}{ Rubro }} & \multicolumn{3}{c}{ Calificación } \\
\cline { 2 - 4 } & HCYA & FCA & MS \\
\hline Calificación global & 7.4 & 7.5 & 6.8 \\
\hline Eje A: Desempeño práctico o funcional & 8.0 & 8.3 & 6.9 \\
\hline Criterio A.1. Eficacia & 9.2 & 9.4 & 8.1 \\
\hline Criterio A.2. Eficiencia & 5.0 & 5.8 & 4.0 \\
\hline Eje B: Desempeño organizativo & 7.6 & 7.3 & 6.6 \\
\hline Criterio B.1. Reglas de decisión & 6.0 & 7.0 & 6.1 \\
\hline Criterio B.2. Procedimientos y mecanismos de decisión & 8.8 & 7.9 & 6.7 \\
\hline Criterio B.3. Integración & 9.5 & 9.3 & 7.8 \\
\hline Criterio B.4. Dirigencia & 7.8 & 6.8 & 6.6 \\
\hline Criterio B.5. Medios de observancia & 7.4 & 7.4 & 7.5 \\
\hline Eje C: Relación con el entorno & 6.9 & 7.3 & 7.0 \\
\hline Criterio C.1 Disponibilidad de capital & 7.7 & 8.0 & 7.5 \\
\hline Criterio C.2. Estrategias frente al desafío... & 3.8 & 7.9 & 6.2 \\
\hline Criterio C.3. Interacción Política & 6.7 & 8.8 & 6.7 \\
\hline Criterio C.4. Representación & 5.0 & 2.5 & 6.7 \\
\hline Criterio C.5. Relevancia social & 5.2 & 4.8 & 5.6 \\
\hline Criterio C.6. Legitimidad & 6.8 & 5.9 & 6.8 \\
\hline
\end{tabular}

Fuente: Elaboración propia con base en los resultados obtenidos de cuestionarios y fichas. 


\section{Gráfico 1}

\section{Desempeño de Marie Stopes}

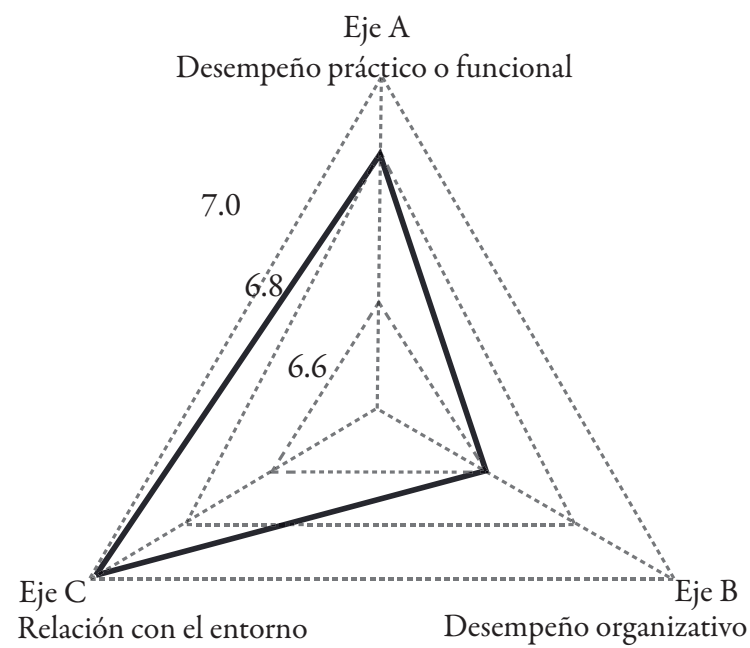

Fuente: Elaboración propia con base en datos del Cuestionario para la Evaluación del Desempeño asociativo.

\section{Gráfico 2}

\section{Desempeño de Fundación Cántaro Azul A.C.}

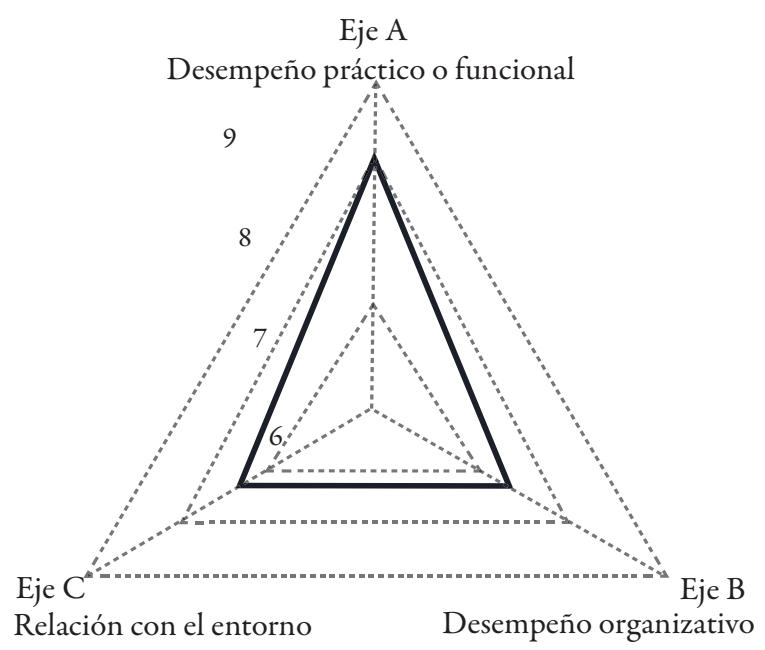

Fuente: Elaboración propia con base en datos del Cuestionario para la Evaluación del Desempeño asociativo. 


\section{Gráfico 3}

\section{Desempeño de Hogar comunitario \\ Eje A}

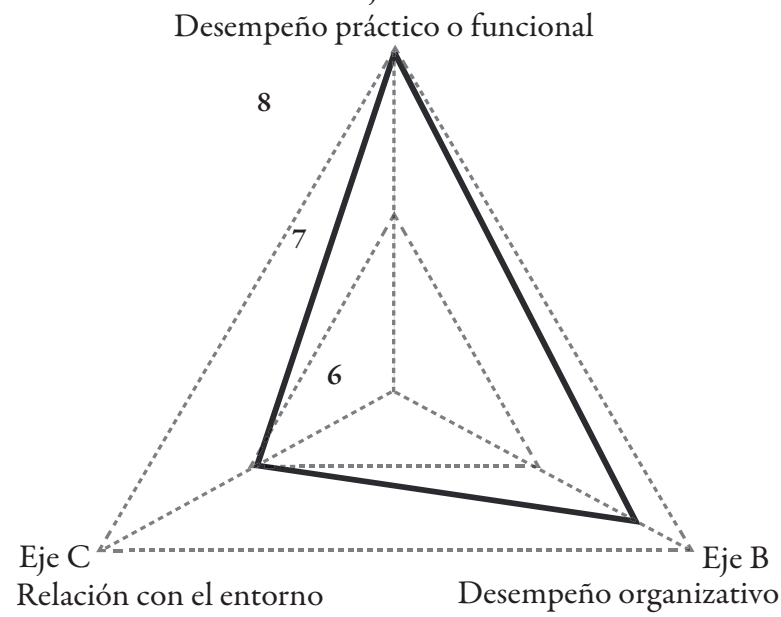

Fuente: Elaboración propia con base en datos del Cuestionario para la Evaluación del Desempeño asociativo.

Gloria Jovita Guadarrama-Sánchez. Doctora en Ciencias Políticas y Sociales por la UNAM, Profesora-Investigadora en El Colegio Mexiquense y miembro del Sistema Nacional de Investigadores, nivel II. Líneas de investigación: instituciones, políticas públicas y sociedad civil. Publicaciones recientes: Un lugar Indefinido: el género en la agenda municipal. La reconstrucción local de la politica nacional en el Estado de México, México: El Colegio Mexiquense (2015); Politicas educativas y agenda de gobierno. Equidady calidad pendientes, México: El Colegio Mexiquense (2012).

Esmeralda Pliego-Alvarado. Estudiante del Doctorado en Ciencias Sociales en la Universidad Autónoma del Estado de México (UAEM), Maestra en Ciencias Sociales con especialidad en desarrollo municipal (2011) por El Colegio Mexiquense, Licenciada en Sociología (2007) por la Universidad Autónoma del Estado de México (UAEM). Ha participado activamente en colectivos y organizaciones civiles como El Grupo Cultural Águila (2000) y Andanza Colectiva (2014). Líneas de investigación: gestión local del agua, estudios socioterritoriales y sociedad civil.

Recepción: 28 de abril de 2016.

Aprobación: 31 de enero de 2017. 
